\title{
Assessment of cardiovascular fitness for competitive sport in high risk groups
}

Regular exercise has been well established to be of physical and psychological benefit to the growing child. Recently, the issue of sudden death during sport has been brought to public attention after the tragic deaths of a few high profile athletes. The need for screening before participation has been queried and task forces have been established to devise recommendations in this area. Physicians are also being faced with a growing population of children and young adults with complex congenital heart defects who are surviving thanks to advances in medical and surgical treatment. An understanding of the residual haemodynamic lesions in these children is essential in order to make sensible recommendations for their ongoing participation in sport. The role of the physician is to impose sensible restrictions based on an assessment of the patient's potential risk of sudden death and of deterioration of cardiac haemodynamics as a result of sport while at the same time encouraging participation in sport as much as is safely possible.

\section{Benefits of exercise}

In adults, regular exercise retards the development of coronary artery disease, hypertension, and obesity. ${ }^{1}$ Training improves cardiac output and oxygen extraction at the tissue level in addition to decreasing myocardial oxygen demands for the same level of external work. ${ }^{2}$ Exercise has also been shown to have psychological benefits and reduce the incidence of stress and depression. These findings are relevant to paediatrics as those who establish regular exercise patterns are more likely to continue these endeavours throughout adulthood. ${ }^{3}$ Participation in sports is an important part of social development for every child and unnecessary restriction can result in alienation and stigmatisation. Children with congenital heart disease can also benefit from regular exercise with an improvement in cardiac output, oxygen consumption, and treadmill times. ${ }^{45}$

Based on this information, some degree of regular exercise should be encouraged in all children but it is up to the paediatric cardiologist to provide safe limits for participation.

\section{Causes of sudden death in young athletes}

In contrast to the older adult population, where coronary artery disease is the predominant cause of sudden death, ${ }^{6}$ children usually die because of unrecognised structural congenital heart disease or electrophysiological abnormalities. The first international study examining this issue in 1974 found that the majority of patients studied either had congenital aortic stenosis, unpalliated cyanotic congenital heart disease, or Eisenmenger's syndrome. ${ }^{7}$ In the current era, these conditions are either dealt with or prevented by early diagnosis and surgical intervention. More recently, Maron et al reviewed a cohort of 29 highly conditioned, competitive athletes between the ages of 13 and 30 who died suddenly. ${ }^{8}$ Within this group, 22 of the 29 died during or immediately after severe exertion and $97 \%$ of these individuals were found to have structural cardiac abnormalities at necropsy. Hypertrophic cardiomyopathy (HOCM) was the most common abnormality (48\%), followed by coronary artery anomalies (14\%), most commonly left coronary artery arising from the right sinus of Valsalva. Rarer causes included premature atherosclerosis $(10 \%)$ and aortic rupture associated with Marfan's syndrome $(7 \%)$. Their findings were confirmed by a subsequent larger multicentre study which demonstrated similar aetiologies. ${ }^{9}$ Silka et al have identified that in their population of postoperative congenital heart patients, those with previous tetralogy of Fallot, aortic stenosis, coarctation, and repairs of transposition of the great arteries were at increased risk for sudden death over their general group. ${ }^{10}$

Electrophysiological abnormalities are being increasingly recognised as potential causes of sudden death in children. In northern England, Wren et al have recently examined all cases of paediatric sudden death between 1985 and 1992 and have found long QT syndrome, unpaced AV block and arrhythmias after cardiac surgery to be more significant causes than undiagnosed HOCM. ${ }^{11}$

\section{Preparticipation screening}

The goal of an effective screening programme should be to identify patients at risk for significant cardiac events during strenuous exercise as non-invasively and cost effectively as possible. Although each event is devastating in its own right, the overall incidence of athletic field death has been estimated between 1:100 000 and 1:300 000 and only $0.2 \%$ of the average athletic population has the various cardiac lesions listed above. ${ }^{12}$ Currently, there is no accepted standardised approach to screening. Financial and technical limitations restrict the implementation of comprehensive screening in most countries.

\section{Components of screening}

HISTORY AND PHYSICAL EXAMINATION

These two modalities alone will not pick up many cardiac causes of sudden death but they do form an essential starting point. ${ }^{13}$ Important points in the history include symptoms of chest pain, syncope or palpitations on exertion, past history of cardiac disease and previous interventions, personal history of coronary risk factors, and a family history of cardiac disease or sudden death before the age of 40. The physical examination should include blood pressure measurement and precordial auscultation for possible left ventricular outflow obstruction (aortic stenosis, HOCM), assessment of the femoral pulses to rule out coarctation of the aorta, and assessment of the body habitus to screen for Marfan's syndrome.

12 LEAD ELECTROCARDIOGRAPHY (ECG)

This is a relatively simple, inexpensive test which may identify patients with HOCM $(95 \%$ of these patients will have abnormal baseline $\mathrm{ECG}^{14}$ ), coronary artery anomalies, long QT syndrome, and certain arrhythmias or risk factors for arrhythmia (for example ventricular preexcitation). Unfortunately, resting ECG has relatively low sensitivity (for example coronary artery anomalies may only produce ischaemic changes on exertion and some genetically affected relatives in families with long QT 
syndrome may have normal ECG). There is also low specificity as ECG abnormalities may be seen in association with adaptations of the normal heart to athletic training. ${ }^{15}$ Nevertheless, this diagnostic tool is readily available and should be considered in individuals planning high intensity competitive sport as a screen to identify those who warrant further cardiac work-up.

\section{ECHOCARDIOGRAPHY}

Patients with concerns or abnormalities noted on routine history, examination, or ECG should undergo specialist cardiac evaluation including echocardiography. This will successfully identify left ventricular outflow obstruction, most cases of HOCM, cardiomyopathy, and aortic root dilatation (for example, Marfan's syndrome). The suspicion of coronary artery anomalies may be raised depending on the quality of imaging. Once again, there are limitations in specificity as the normal 'athletic heart' may have echocardiographic changes which must be differentiated from pathological changes. ${ }^{16}$

\section{EXERCISE TESTING}

Exercise testing in the otherwise healthy athlete should be limited to those with exercise induced symptoms or proved underlying structural or arrhythmic abnormalities. Ideally the design and intensity of the test should be tailored to approximate the nature of the planned athletic activity.

\section{Other modalities}

Holter monitoring, electrophysiology studies, and angiography are tools available to the paediatric cardiologist that should be reserved for specific cases where life threatening arrhythmias or coronary anomalies are strongly suspected.

It is possible that in the near future, genetic screening will become available for conditions such as HOCM, Marfan's syndrome, and long QT syndrome. However, present knowledge indicates that the genetic mutations are quite heterogeneous, particularly from one family to another, making mass screening impractical. Furthermore, there may be significant variation in phenotypic expression making postscreening counselling difficult even when genetic defects have been identified.

\section{Perspectives on screening}

The large numbers of children participating in competitive sport makes universal screening economically non-viable. Furthermore, even if screening is employed not all cardiac problems are detected with certainty. False positive results may result in a child being inappropriately restricted from participation. A unique perspective on screening can be gained by examining the situation in Italy where preparticipation screening has been in place for three decades. ${ }^{17}$ All elite athletes are subjected to a medical examination, 12 lead ECG, exercise testing, and echocardiography. Since $1963,2.2 \%$ of these athletes have been restricted from competition because of relevant cardiovascular abnormalities and as a result, HOCM, coronary artery anomalies, and aortic root dissection form a much smaller percentage of field deaths. It is not clear, however, whether the overall incidence of field death has been reduced by such a screening programme. It is reasonable however to recommend that all children planning intense physical activity should at least undergo a complete medical examination with further investigations carried out where appropriate. Careful deliberation must be made before restrictions are placed.

\section{Sports participation guidelines in paediatric cardiac disease}

All children with established cardiac disease need evaluation by a primary care physician and paediatric cardiologist to assess the nature of the underlying lesion, interventions (surgical, medical) received, and residual haemodynamic disturbances. ${ }^{18}{ }^{19}$ The nature of the planned sport needs to be assessed for its degree of static versus dynamic work, the danger of bodily collision, and potential danger if sudden incapacitation or syncope were to occur. The 26th Bethesda Conference held in 1994 established recommendations for various cardiac lesions and serves as a useful reference. ${ }^{20}$

\section{Types of lesion}

ACYANOTIC LESIONS ASSOCIATED WITH VOLUME LOADING Acyanotic lesions associated with volume loading (atrial or ventricular septal defects, patent ductus arteriosus, or valvular regurgitation) are generally accompanied by good exercise tolerance unless they are severe, in which case intervention is usually warranted. No limitations are necessary unless there is associated left ventricular dysfunction, pulmonary vascular disease (unusual in the present era as a result of early surgical intervention), or symptomatic arrhythmias. Patients with valvular regurgitation necessitating prosthetic valve replacement are kept anticoagulated and should, therefore, avoid all sports with potential bodily contact.

\section{ACYANOTIC LESIONS ASSOCIATED WITH OBSTRUCTION}

Pulmonary stenosis does not require any restriction unless it is moderately severe (that is gradient $>40 \mathrm{~mm} \mathrm{Hg}$ ) in which case balloon valvuloplasty is usually indicated. Exercise can be resumed once the gradient has been successfully reduced and there is a very low incidence of restenosis.

Severe aortic stenosis is associated with a risk of sudden death especially when there is associated left ventricular hypertrophy with strain. As a general rule, patients with mild aortic stenosis (gradient $<20 \mathrm{~mm} \mathrm{Hg}$, normal ECG, and no symptoms) do not require restriction. Patients with moderate aortic stenosis $(20-50 \mathrm{~mm} \mathrm{Hg}$, mild left ventricular hypertrophy without strain on ECG, and no symptoms) should undergo exercise testing and if this is normal can be allowed to participate in competitive low to moderate intensity sports. Patients with severe aortic stenosis should be restricted from all competitive sports until they have had appropriate intervention. In particular, high intensity static pursuits such as weight lifting are extremely dangerous as they increase afterload. Even after intervention, regular long term follow up is important as progressive restenosis or regurgitation may develop.

Similar recommendations apply for coarctation of the aorta with the added potential problem of exercise induced hypertension both before and after intervention. This should be treated before active sports are commenced.

Patients with hypertrophic obstructive cardiomyopathy have an increased risk of sudden death with exertion and should therefore be restricted from all competitive sports. ${ }^{17}$ Low intensity recreational sport can only be undertaken if there is mild left ventricular hypertrophy and no history of arrhythmias.

CYANOTIC LESIONS

Children with cyanotic lesions generally have a limited exercise capacity for a variety of reasons. Exercise reduces systemic vascular resistance which can worsen a preexisting right to left shunt. These patients usually have limited pulmonary blood flow and therefore cannot increase it as is required during exercise. Chronic cyanosis promotes polycythaemia and hyperviscosity which in turn impairs oxygen delivery at the tissue level.

Children who have undergone surgical correction of tetralogy of Fallot usually have good exercise tolerance 
unless there are significant residual defects (for example, ventricular septal defects, pulmonary regurgitation). Although important ventricular arrhythmias can be seen postoperatively, they are rare. Therefore, this group of children should be allowed to exercise without limitation unless they have been shown to have reproducible exercise induced ventricular tachycardia or significant right ventricular dysfunction.

Before the introduction of the arterial switch, intra-atrial repair (Mustard or Senning procedure) was the standard operation for transposition of the great arteries and many patients have survived to adolescence and adult life. Most of these patients have good exercise tolerance. Unless there is ventricular dysfunction, baffle obstruction, arrhythmias or atrioventricular valve regurgitation, no specific limitations need to be placed. There is, however, a worrying incidence of late sudden death but currently, identification of high risk subjects is difficult. The follow up to date of arterial switch patients suggests that these patients have excellent exercise tolerance. Avoidance of heavy isometric exercise (weightlifting) has been suggested because of the potential risk of aggravating neoaortic regurgitation. Coronary anomalies have also been reported but their clinical significance is not yet clear.

The third important group of postoperative cyanotic heart patients is those children who have undergone the Fontan procedure (or one of its variations), which essentially separates systemic and pulmonary venous return by routing systemic venous blood directly to the pulmonary arteries. It is employed in the palliation of various forms of severe right heart obstruction and circulations with a single ventricular chamber. Pulmonary blood flow occurs passively with no right ventricular input and, as a result, these patients have limited cardiac output and reduced peak exercise ability. After a successful Fontan procedure, however, performance of moderate exercise may be improved and, thus, these children should be encouraged to perform as much exercise as they can comfortably tolerate.

\section{MISCELLANEOUS LESIONS}

Marfan's syndrome

These patients need serial echocardiographic assessments to screen for aortic root dilatation. No restrictions are required if the aortic root dimensions are normal. If aortic root dilatation is present, patients should be restricted to low intensity sports where there is no risk of bodily collision. Serial assessments are crucial as haemodynamic deterioration may be especially evident during adolescence.

\section{Kawasaki disease}

Patients with this condition and no evidence of coronary artery involvement on echocardiography can exercise without limitation. Those with minor residual abnormalities can participate in mild to moderate dynamic exercise and those with persistent aneurysms or stenosis should probably be restricted from competitive sport.

\section{Role of the physician}

The physician must act both as the child's counsellor and advocate. This involves a thorough assessment of the patient's cardiovascular status and the potential risks of participation. Recommendations need to be discussed with the patient and family and communicated clearly to others involved with the child such as school teachers. Emphasis should be placed on involving these children as much as is safely allowable as there may often be an automatic assumption that the child should be restricted. It must be remembered that cardiovascular fitness is not a static entity and should be reviewed on an ongoing basis.

\section{Conclusion}

In summary, participation in sport forms an integral part of childhood and should be encouraged whenever possible. Sudden death during exercise is extremely rare in this age group and is most often due to undetected structural congenital heart disease. All children contemplating intense competitive sport should ideally have a medical examination and, if possible, ECG. Referral to a paediatric cardiologist for further assessment is indicated if the initial evaluation uncovers any concerns. Exercise should be encouraged in children with congenital heart disease within the limits of their residual lesions. Although useful general guidelines exist, each patient must be evaluated on an individual basis with ongoing regular reassessments. Accumulating knowledge will enable rational decision making in this important area.

N R SONI

J E DEANFIELD

Cardiothoracic Unit

Great Ormond Street Hospital for Children,

London WC1N $37 H$

1 Roccini AP, Katch V, Anderson J, et al. Blood pressure in obese adolescents: effect of weight loss. Pediatrics 1988;82:16-23.

2 Fletcher GF, Balady G, Blair SN, et al. Statement of exercise: benefits and recommendations for physical activity programs for all America. Circulation 1996;94:857-62.

3 Committee on Sports Medicine and Fitness. Assessing physical activity and fitness in the office setting. Pediatrics 1994;93:686-9.

4 Goldberg B, Fripp RR, Lister G, et al. Effect of physical training on exercise performance of children following surgical repair of congenital heart disease. Pediatrics 1981;68:691-9.

5 Ruttenberg HD, Adams TD, Orsmond GS, et al. Effects of exercise training on aerobic fitness in children after open heart surgery. Pediatr Cardiol 1983; 4:19-24

6 Waller BF, Roberts WC. Sudden death while running in conditioned runners aged 40 years and over. Am f Cardiol 1980;45:1292-300.

7 Lambert EC, Menon VA, Wagner HR, et al. Sudden unexpected death from cardiovascular disease in children. Am f Cardiol 1974;34:89-96.

8 Maron BJ, Roberts WC, McAllister MD. Sudden death in young athletes. Circulation 1980;62:218-29.

9 Maron BJ, Epstein SE, Roberts WC. Causes of sudden death in competitive Maron BJ, Epstein SE, Roberts WC. Causes
athletes. $¥$ Am Coll Cardiol 1986;7:204-14.

10 Silka MJ, Hardy BD, Menashe VD, et al. A population-based prospective study of sudden, unexpected cardiac death following surgery for common congenital heart defects: implications for design and future evaluation of this problem (abstract from NASPE, 1997). Pacing Clin Electrophysiol 1997; 20:1051.

11 Wren C, O'Sullivan JJ, Wright C. Sudden death in children and adolescents - a population based study (abstract from NASPE, 1997). Pacing Clin Electrophysiol 1997;20:1051.

12 Maron BJ, Thompson PD, Puffer JC, et al. Cardiovascular preparticipation screening of competitive athletes. Circulation 1996;94:850-6.

13 Goodwin JF. Sudden cardiac death in the young. BMF 1997;314:843.

14 Maron BJ, Roberts WC, Epstein SE. Sudden death in hypertrophic cardiomyopathy: a profile of 78 patients. Circulation 1982;65:1388-94.

15 Zehender M, Meinerts T, Keul J, et al. ECG variants and cardiac arrhythmias in athletes: Clinical relevance and prognostic importance. $A m$ Heart $\mathcal{F}$ 1990;119:1378-9.1

16 Maron BJ. Structural features of the athlete heart as defined by echocardiography. F Am Coll Cardiol 1986;7:190-203.

17 Pelliccia A, Maron BJ. Preparticipation cardiovascular evaluation of the competitive athlete: perspectives from the 30 year Italian experience. $\mathrm{Am} \mathcal{F}$ Cardiol 1995;75:827-9.

18 Cullen S, Celermajer DS, Deanfield JE. Exercise in congenital heart disease. Cardiol Young 1991;1:129-35.

19 Fratellone PM, Steinfeld L, Coplan NL. Exercise and congenital heart disease. Am Heart f 1994;127:1676-80

20 Maron BJ, Mitchell JH. 26th Bethesda conference: recommendations for determining eligibility for competition in athletes with cardiovascular abnormalities. F Am Coll Cardiol 1994;24: 845-99. 